
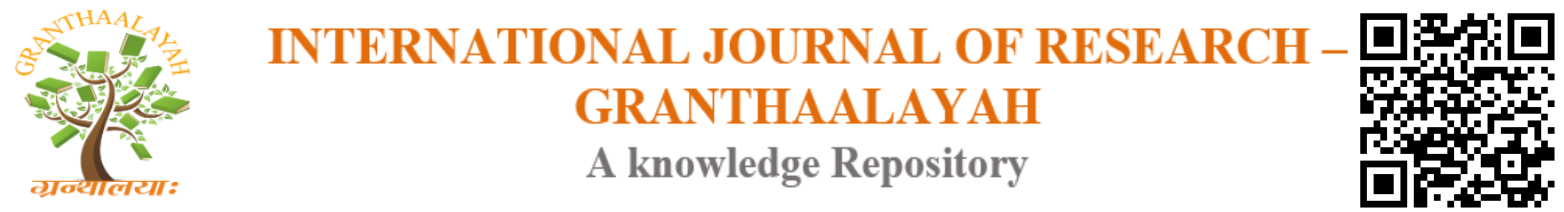

Science

\title{
FIGHTING AGAINST GLOBAL HIV EPIDEMIC: AVAILABLE OPTIONS TO FOCUS ON
}

\author{
Homa Nath Sharma 'Raju' *1, Sagar Baral ${ }^{2}$, Yadav Baral ${ }^{3}$ \\ ${ }^{* 1}$ M.Sc. Graduate, Central Department of Microbiology, TU, Kirtipur, Kathmandu, Nepal, \\ Science Teacher, Gandhi Adarsha Secondary, School, Kandaghari, Kathmandu, Nepal, \\ Prospective (Starting on Sep 4, 2019) Ph.D. Student, University of Wyoming, Laramie, WY, \\ USA \\ ${ }^{2}$ M.B.B.S. Graduate, Institute of Medicine, TU Teaching Hospital, Maharajgunj, Kathmandu, \\ Nepal Medical Officer, Parbat District Hospital, Kusma, Parbat, Nepal \\ ${ }^{3}$ Undergraduate Student, University of Texas at Arlington, TX, USA
}

\begin{abstract}
HIV continues to be a major global public health issue, having claimed more than 32 million lives so far. While great progress has been made in preventing and treating HIV, there is still much to do. A safe and cost-effective vaccine that prevents HIV infection is considered the best strategy for containing the epidemic. However; historically, the vaccine development for HIV prevention has been extremely difficult. Anti-Retroviral Therapy (ART) has improved the quality of life of Human immunodeficiency virus (HIV) patients worldwide. This reality suggests that continuing on HIV vaccine research, now we should also consider applying other short-term available prevention and treatment strategies to mitigate the effect of the epidemic.
\end{abstract}

Keywords: HIV; Public Health; HIV Vaccine.

Cite This Article: Homa Nath Sharma 'Raju', Sagar Baral, and Yadav Baral. (2019). "FIGHTING AGAINST GLOBAL HIV EPIDEMIC: AVAILABLE OPTIONS TO FOCUS ON." International Journal of Research - Granthaalayah, 7(7), 102-105.

\section{Introduction}

HIV continues to be a major global public health issue, having claimed more than 32 million lives so far. In 2018, 770000 people died from HIV-related causes globally (1).About 37.9 million people were living with HIV around the world in 2018. Worldwide, there were about 1.7 million new cases of HIV in 2018 (2) as compared to 1.8 million in 2017 (3). New HIV infections have been reduced by $40 \%$ since the peak in 1997 and AIDS-related mortality has declined by $33 \%$ since 2010 (2).While great progress has been made in preventing and treating HIV, there is still much to do (4). 
A safe and cost-effective vaccine that prevents HIV infection is considered the best strategy for containing the epidemic (5). However; historically, the vaccine development for HIV prevention has been extremely difficult (6). 30 years of HIV vaccine research has wasted, with an exception to modest $31 \%$ efficacy of RV 144 trial (7). It is partly due to the fact that the process of vaccine development typically takes a long time period, partly to the failure of classical viral vaccination strategies when applied to HIV and partly to the many barriers to immune recognition evolved by the virus (8) No understood natural protective immunity to HIV infection and disease, and HIV genome's capability of profound and durable variability confounding typical host's immune response are two challenges for HIV vaccine development. (9). Development of an HIV vaccine is a winding challenge and the research on this will require long-term commitment $(10,11)$. So even if a safe and effective vaccine were developed and disseminated, which is still 5-10 years away (12), the primary challenge will be supporting uptake by consumers to reach adequate population coverage (13). This reality suggests that continuing on HIV vaccine research, now we should also consider applying other short-term available prevention and treatment strategies to mitigate the effect of the epidemic.

ART has improved the quality of life of HIV patients worldwide. A reduction in HIV-related morbidity and mortality has been recognized in countries where ART has been made widely available. Acquired immune deficiency syndrome (AIDS) is now a manageable chronic illness (14). When taken consistently as prescribed, ART can suppress viral load, maintain high CD4 cell counts, prevent AIDS, prolong survival, and reduce risk of transmitting HIV to others, all of which are important treatment goals. $(15,16)$. It is also to note that the life expectancy in HIV-positive people on ART has improved worldwide in recent years (17). Between 2000 and 2018, HIV-related deaths fell by $45 \%$ with 13.6 million lives saved due to ART in the same period. But only62\% of adults and 54\% of children living with HIV were receiving lifelong ART in 2018, while only 79\% of people with HIV know their status (1). Additionally, despite the success of standard regimens, ART is complicated by potential drug-drug interactions, patient non-adherence and adverse effects (5). It is easily projected that providing health education and healthcare to all people in the world can include all people with HIV on board for letting them their status so that no one may be deprived of ART. Alcohol use, drug side effects, depression, stigma and lack of family support are factors associated with reduced adherence of PLHA to ART. Priorities should be given to improving adherence otherwise our ART program will fail (18). So, extensive research on these areas, smooth delivery and regular monitoring of available services to all levels, as well as relevant improvements seem need of hour.

Vulnerable population of HIV like victims of nonconsensual high-risk sexual activities are in need of aggressive preventive approach in terms of pre- and post-sexual exposure prophylaxis- PrEP and PEP (19). PrEP is of substantial benefit in decreasing the risk of HIV infection in persons at high risk of HIV acquisition. Adherence to PrEP is highly correlated with its efficacy in preventing the acquisition of HIV infection (20).

Although there have been major accomplishments in HIV chemotherapy, since drug resistance to anti-HIV drugs is emerging, and many people infected with HIV have adverse reactions or do not have ready access to currently available HIV chemotherapies, there remains a need for new antiHIV drug discovery, and medicinal plants can play an important role in this endeavor (21). There are many herbal remedies that have been found to inhibit one or more steps in HIV replication (22, 
23). In particular, in vivo testing and, ultimately, human clinical trials need to be carried out on key lead plants and phytochemical isolates. In addition, continuous evaluation of medicinal plants for anti-HIV activity should be pursued (21).

Pitfalls in current HIV prevention strategies include late HIV testing, vulnerability among youth and females; lack of emphasis on treatment and low acceptance of circumcision in addition to the no availability of protective vaccines (19). Out of them, first three are technical issues to resolve. Since circumcision is only $60 \%$ protective against HIV acquisition, it is possible —or even probable - that similar mechanisms underpin their residual HIV susceptibility, as well as HIV susceptibility in women. Therefore, new modalities to reduce genital immune activation and/or alter the genital microbiome, used alone or in combination with topical microbicides, may be of significant benefit in HIV prevention (24).

Regarding the ultimate long-term obstacle to overcome, currently, about 30 NIAID -sponsored preventive HIV vaccine clinical trials are underway or planned for various stages of testing in the various part of world. In the next 2 years, more vaccines will be studied than in the last 5 years combined. Although the challenges for scientist are intimidating but scientists remain hopeful that they can develop safe and effective HIV vaccines for patients in future (25).To date, it has been found that broadly neutralizing antibodies, or bNAbs, can stop many HIV strains from infecting human cells in the laboratory (26). Clinical trials and preclinical proof-of-concept studies in nonhuman primate (NHP) models have also provided insights into potential correlates of protection against the HIV-1 infection, which include broadly neutralizing antibodies (bnAbs), non-neutralizing antibodies targeting the variable loops 1 and 2 (V1V2) regions of the HIV1envelope (Env), polyfunctional antibody, and Env-specific T-cell responses (27). Latter two momentums might turn out the current pessimism into optimism for humanity that HIV vaccine would be the reality to control and even eradicate the global HIV epidemic.

\section{References}

[1] World Health Organization (WHO). HIV/AIDS: Key facts. www.who.int/news-room/factsheets/detail/hiv-aids/. Accessed 17 July, 2019.

[2] United Nations Programme on HIV and AIDS (UNAIDS). Global HIV and AIDS statistics-2019 fact-sheets, www.unaids.org/en/resources/fact-sheet. Accessed July 7, 2019.

[3] United Nations Programme on HIV and AIDS (UNAIDS). UNAIDS data 2018.www.unaids.org. Accessed July 16, 2018.

[4] Center for Disease Control and Prevention (CDC). HIV/AIDS: Basic Statistics.www.cdc.gov/hiv/HIVbasic/statistics/. Accessed April 12, 2019

[5] Bossou A R, Castrillo S, O’Brien K K, et al. Preventive HIV Vaccine: Progress and Challenges. US Pharm. 2015; 40(10):46-50.

[6] Collins F. Vaccine Research: New Tactics for Tackling HIV. http://directorsb/og.nih.gov/2015/06/30/vaccine-research-newtactics-for-tackling-hiv. Accessed September 10, 2015.

[7] Shin Y. Recent Update in HIV Vaccine Development. Clin Exp Vaccine Res. 2016; 5:6-11. http://dx.doi.org/10.7774/cevr.2016.5.1.6.

[8] Burton DR, Ahmed R, Barouch DH, et al. A Blueprint for HIV Vaccine Discovery. Cell Host Microbe. 2012; 12(4):396-407. DOI:10.1016/j.chom.2012.09.008.

[9] Brett-Major D M, Crowell T A, Michael N L. Prospecting for an HIV Vaccine. Tropical Diseases, Travel Medicine and Vaccines. 2017; 3:6. DOI: 10.1186/s40794-017-0050-4. 
[10] Esparza J. What Has 30 Years of HIV Vaccine Research Taught Us? Vaccines (Basel). 2013; 1(4):513-526. Published 2013 Oct 30. DOI:10.3390/vaccines 1040513

[11] Shin SY. Recent Update in HIV Vaccine Development. Clin Exp Vaccine Res. 2016; 5(1): 6-11. DOI:10.7774/cevr.2016.5.1.6

[12] Girard MP, Osmanov SK, Kieny MP. A Review of Vaccine Research and Revelopment: The Human Immunodeficiency Virus (HIV) Vaccine. 2006;24(19):4062-4081.

[13] Newman PA, Duan N, Rudy ET, Anton PA. Challenges for HIV Vaccine Dissemination and Clinical Trial Recruitment: If We Build It, Will They Come? AIDS Patient Care STDs. 2004a; 18(12):691-701.

[14] Paterson DL, Swindells S, Mohr J, et al. Adherence to Protease Inhibitor Therapy and Outcomes in Patients with HIV Infection. Ann Intern Med. 2000;133:21-30.

[15] Garcia F, de Lazzari E, Plana M, et al. Long-term CD4+ T-cell Response to Highly Active Antiretroviral Therapy According to Baseline CD4+ T-cell Count. J Acquir Immune Defic Syndr. Jun 1 2004;36(2):702-713.

[16] Cohen MS. Prevention of HIV-1 Infection with Early Antiretroviral Therapy. N Engl J Med 2011; 365(6):493-505.

[17] Wandeler G, Johnson LF, Egger M. Trends in Life Expectancy of HIV-positive Adults on Antiretroviral Therapy Across the Globe: Comparisons with General Population. Curr Opin HIV AIDS. 2016; 11(5):492-500. DOI:10.1097/COH.0000000000000298.

[18] Achappa B, Madi D, Bhaskaran U, et al. Adherence to Antiretroviral Therapy Among People Living with HIV. N Am J Med Sci. 2013;5(3):220-223. DOI:10.4103/1947-2714.109196.

[19] Marfatia YS, Jose SK, Baxi RR, Shah RJ. Pre- and Post-sexual Exposure Prophylaxis of HIV: An update. Indian J Sex Transm Dis AIDS. 2017;38(1): 1-9. DOI:10.4103/ijstd.IJSTD_26_17

[20] US Preventive Service Task Force. Recommendation Statement. Pre-exposure Prophylaxis on HIV Infection.

JAMA.2019;321(22):2203-2213. https://www.uspreventiveservicestaskforce.org/Page/Name/recommendations. DOI:10.1001/jama.2019.6390.

[21] Salehi B, Kumar NVA, Şener B, et al. Medicinal Plants Used in the Treatment of Human Immunodeficiency Virus. Int J Mol Sci. 2018;19(5):1459. Published 2018 May 14. DOI 10.3390/ijms 19051459

[22] De Clereq. Current Lead Natural Products for the Chemotherapy of Human Immunodeficiency Virus Infection. Med Res Rev. 2000;20:323-349.

[23] Kong J M, Goh N K, Chia L S, Chia T F. Recent Advances in Traditional Plant Drugs and Orchids. Acta Pharmacol Sin. 2003;24:7-21.

[24] Prodger J L, Kaul R. The Biology of How Circumcision Reduces HIV Susceptibility: Broader Implications for the Prevention Field. AIDS Res Ther. 2017;14:49. DOI: 10.1186/s12981-0170167-6.

[25] Ramesh Verma, Pardeep Khanna, Suraj Chawla \& Mukesh Dhankar (2016) HIV vaccine: Can it be developed in the 21st century? Human Vaccines \& Immunotherapeutics. 2016; 12(1): 222-224. DOI: 10.1080/21645515.2015.1064571.

[26] National Institute of Allergy and Infectious Diseases (NIAID). Progress toward an HIV Vaccine. www.niaid.nih.gov. Accessed July 7, 2019

[27] Trovato M, D'Apice L, Prisco A, et al. HIV vaccine: A Roadmap among Advancements and Concerns. Int J Mol Scssi. 2018; 19: 1241. DOI: 10.3390/ijms19041241.

\footnotetext{
*Corresponding author.
}

E-mail address: hnsharmaraju99@gmail.com/sagarbaral2012mbbs@gmail.com/baral.yadav@ yahoo.com 